\title{
MULTIPLE BUYING OR SELLING WITH VECTOR OFFERS
}

\author{
F. THOMAS BRUSS*, Université Libre de Bruxelles \\ THOMAS S. FERGUSON**, University of California, Los Angeles
}

\begin{abstract}
We consider a generalization of the house-selling problem to selling $k$ houses. Let the offers, $\mathbf{X}_{1}, \mathbf{X}_{2}, \ldots$, be independent, identically distributed $k$-dimensional random vectors having a known distribution with finite second moments. The decision maker is to choose simultaneously $k$ stopping rules, $N_{1}, \ldots, N_{k}$, one for each component. The payoff is the sum over $j$ of the $j$ th component of $\mathbf{X}_{N_{j}}$ minus a constant cost per observation until all stopping rules have stopped. Simple descriptions of the optimal rules are found. Extension is made to problems with recall of past offers and to problems with a discount.
\end{abstract}

KEYWORDS: OPTIMAL STOPPING, HOUSE-SELLING, JOB SEARCH, COST, DISCOUNT, RECALL, CONVEX SET FUNCTION

AMS 1991 SUBJECT CLASSIFICATION: PRIMARY 60G40

SECONDARY 62L15

$\S 1$. Introduction and Summary. You own $k$ objects. Each day you receive a vector of offers, say $\mathbf{X}_{n}=\left(X_{n}^{(1)}, \ldots, X_{n}^{(k)}\right)$ on day $n$, where $X_{n}^{(j)}$ represents that day's offer for the $j$ th object. It is assumed that $\mathbf{X}_{1}, \mathbf{X}_{2}, \ldots$ are independent and identically distributed having a known $k$-variate distribution with finite second moments. There is a cost of $c>0$ per vector of observations. At each stage you may sell none, any one, any two, ..., or all of the objects. You are to continue until all objects are sold. Your payoff is the sum of the selling prices of the objects minus $c$ times the number of vectors observed.

* Address: Université Libre de Bruxelles, Département de Mathématique et Institut de Statistique, CP 210, B-1050 Brussels, Belgium, e-mail: tbruss@ulb.ac.be

** Address: Department of Mathematics, University of California, Los Angeles, CA 90095, USA, e-mail: tom@math.ucla.edu 
This problem was suggested to us by James B. MacQueen who modeled it as a buying problem. You want to buy Christmas presents for your two children. After deciding which two presents to buy, you go to various stores. With two presents to buy, you can be a little more choosy. If the price of one of the gifts is clearly too high, you know you will have to go to another store anyway, so you will reject a borderline price for the other gift.

This problem is a multidimensional generalization of the celebrated house-selling problem, introduced by MacQueen and Miller (1960), Derman and Sacks (1960), Chow and Robbins (1961), and Sakaguchi (1961). In the economics literature, this problem is known as the job search problem and traced back to Stigler (1961, 1962). See Collins and McNamara $(1990,1993)$ for recent work and McMillan and Rothschild (1994) for a review of this aspect of the problem.

Various multiple house-selling problems have already appeared in the literature. The original paper of MacQueen and Miller (1960) contains an extension to the problem of selling $k$ identical objects when single offers come in daily, and recall of past offers is allowed. The paper of Karlin (1962), contains a discussion of the problem of selling two identical objects with finite horizon and no cost, with one offer per time period and no recall of past offers. This has been generalized by Derman, Lieberman and Ross (1972), Albright (1974) and others (see Righter (1990)) to selling non-identical objects with one offer per time period. It has also been generalized by Saario (1986), Stadje (1985, 1990) and Saario and Sakaguchi (1990) to selling $k$ identical objects with cost, finite horizon and random arrival of offers. The only treatment of house-selling problems with vector offers, seems to be by Sakaguchi (1973 and 1978), but this appears in a game-theoretic setting in which two players must agree to stop and accept the present bivariate offer, and the problem is to find equilibrium points.

In the case of vectors of independent identically distributed offers, one could set up a classical multiple house-selling model with batch arrivals to treat this problem. However, one would need the rather unnatural assumption that the batch sizes be exactly equal to the number of objects remaining to be sold. The closest to this approach in the literature seems to be the paper of Nakai (1986). But in his paper, there is a finite horizon rather than a cost, and the sizes of the batches are random with binomial distributions. 
In Section 2, we treat completely the problem of selling two objects and indicate the effect of dependence of the offers on the optimal return. In Section 3, we treat the general problem and note an interesting convexity property of the value function. In Section 4, we solve the problem when recall of past offers is allowed. In the one object case, there is no difference between the optimal rules for selling with or without recall, as observed by Chow and Robbins (1961). For selling several objects, the rules become quite distinct, and the methods of treatment are different as well. In Section 5, some models in which the cost is replaced by a discount are investigated.

$\S 2$. Selling two objects. We specialize the description of the problem in the introduction to $k=2$ objects and denote the sequence of vector offers by $\left(X_{1}, Y_{1}\right),\left(X_{2}, Y_{2}\right), \ldots$, assumed to be i.i.d. with finite second moments.

Once one object is sold, the problem reduces to the standard house-selling problem. From the general theory, see for example Chow, Robbins and Siegmund (1971), it is known, under the condition that the second moments be finite, that an optimal rule exists and is given by the principle of optimality. Furthermore, the optimality equation holds. Let $V_{x}$ and $V_{y}$ denote the optimal values of the problems of selling the $x$-object and $y$-object separately. These values are the unique solutions of the optimality equations,

$$
\mathrm{E}\left(X-V_{x}\right)^{+}=c \quad \text { and } \quad \mathrm{E}\left(Y-V_{y}\right)^{+}=c .
$$

Theorem 1. In the problem of selling two objects with i.i.d. vector offers having finite second moments, an optimal rule exists and the value, $V_{x y}$, is the unique solution of the equation,

$$
V_{x y}=\mathrm{E} \max \left\{X+Y, X+V_{y}, Y+V_{x}, V_{x y}\right\}-c .
$$

Proof. We frame the problem as a stopping rule problem where stopping is identified with selling at least one object. If we stop at stage $n$ having observed $\left(X_{n}, Y_{n}\right)$, we may choose to sell both objects and receive $X_{n}+Y_{n}$, sell the $x$-object alone and receive $X_{n}+V_{y}$, or sell the $y$-object alone and receive $Y_{n}+V_{x}$. Thus, if we stop at stage n, we receive $W_{n}-n c$, where $W_{n}=\max \left\{X_{n}+Y_{n}, X_{n}+V_{y}, Y_{n}+V_{x}\right\}$. Since the $W_{n}$ are i.i.d. with finite second 
moments, the general theory implies that an optimal rule exists and that $V_{x y}$ is the unique solution to the optimality equation, $V_{x y}=\operatorname{E} \max \left\{W_{1}, V_{x y}\right\}-c$. This reduces to equation $(2)$.

The optimal rule given by the principle of optimality is to continue sampling until $W_{n} \geq V_{x y}$, and then to stop and sell both objects if $X_{n}+Y_{n}=W_{n}$, the $x$-object alone if $X_{n}+V_{y}=W_{n}$, and the $y$-object alone if $Y_{n}+V_{x}=W_{n}$. The following lemma helps us to simplify this description.

Lemma 1. The value function is superadditive, namely, $V_{x y}>V_{x}+V_{y}$.

Proof. $V_{x}+V_{y}$ is the optimal return for selling each of the objects separately. When selling them together, one can use the same individual strategies and even only have to pay one $c$ per observation until the first of them is sold.

In fact this argument shows something stronger, that $V+c$ is superadditive, namely, $V_{x y} \geq V_{x}+V_{y}+c$.

Theorem 2. An optimal rule for the 2-object problem at stage $n$ is as follows. Sell the $x$-object if $X_{n} \geq V_{x y}-V_{y}$. Else, sell the $y$-object if $Y_{n} \geq V_{x y}-V_{x}$. Else, sell both objects if $X_{n}+Y_{n} \geq V_{x y}$. If one object is sold, revert to the optimal strategy for selling the other object before looking at the next observation. Else, continue to stage $n+1$.

Proof. From Lemma 1, the expectation in (2) reduces to the sum of the integrals over the four regions,

$$
\begin{aligned}
& \mathrm{A} 1=\left\{X>V_{x y}-V_{y}, Y<V_{y}\right\} \\
& \mathrm{A} 2=\left\{Y>V_{x y}-V_{x}, X<V_{x}\right\} \\
& \mathrm{A} 3=\left\{X<V_{x y}-V_{y}, Y<V_{x y}-V_{x}, X+Y<V_{x y}\right\} \\
& \mathrm{A} 4=\left\{X>V_{x}, Y>V_{y}, X+Y>V_{x y}\right\} .
\end{aligned}
$$

The rule given by the principle of optimality is to sell both objects in region A4, in region A1 sell the $x$-object only, in region A2 sell the $y$-object only, and sell neither object in region A3 (see Figure 1). Thus, we always sell the $x$-object if $X \geq V_{x y}-V_{x}$. And if this is the case, we sell the $y$-object as well if $Y \geq V_{y}$, which is the optimal strategy for selling 
Figure 1. The optimal regions.

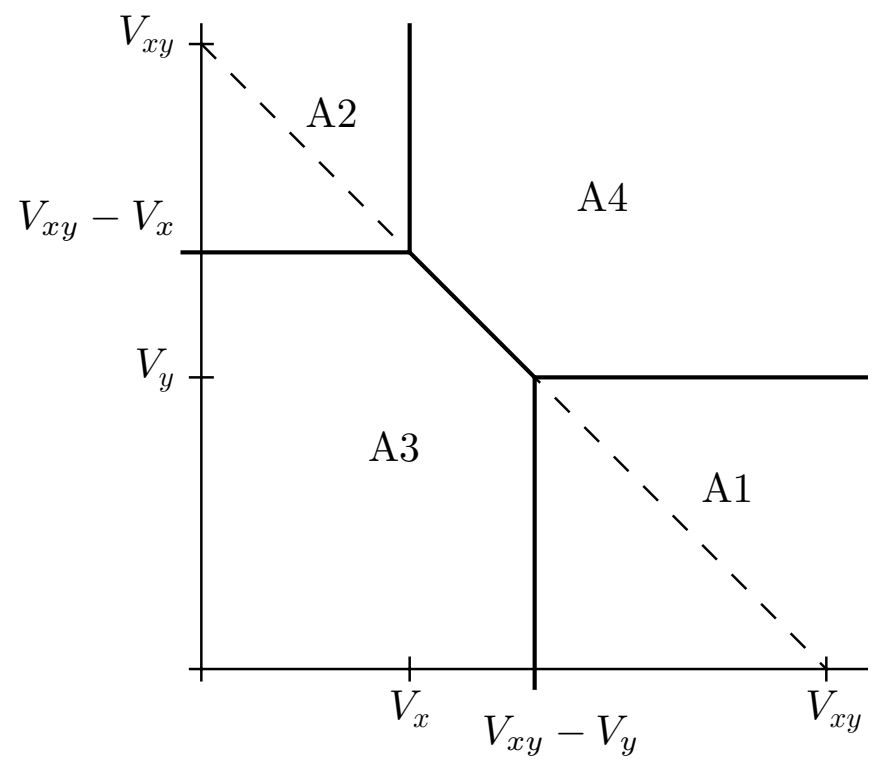

one object. In this way, we may reduce the description of the optimal strategy to that given in the theorem.

$\S 2.1$ Example. Suppose that $X$ and $Y$ are independent and identically distributed according to the uniform distribution on the interval $(0,1)$. Then as found in DeGroot (1970) for example, equations (1) reduce to

$$
V_{x}=V_{y}= \begin{cases}1-\sqrt{2 c} & \text { if } c \leq 1 / 2 \\ -c+(1 / 2) & \text { if } c \geq 1 / 2\end{cases}
$$

Let $V_{1}=V_{x}=V_{y}$. For $0<c \leq 1 / 2$, the respective parts of the integrals in Equation (2) over the four regions become,

$$
\begin{aligned}
& \text { A1 }: V_{1}\left(\left(1+V_{1}\right)^{2}-V_{x y}^{2}\right) / 2 \\
& \text { A2 }: V_{1}\left(\left(1+V_{1}\right)^{2}-V_{x y}^{2}\right) / 2 \\
& \text { A3 }: V_{x y}\left(V_{x y}^{2}-2 V_{1}^{2}\right) / 2 \\
& \text { A } 4:\left(1-V_{1}\right)^{2}\left(1+V_{1}\right)-\left(V_{x y}-2 V_{1}\right)^{2}\left(V_{x y}+V_{1}\right) / 3 .
\end{aligned}
$$

Adding these, we find that equation (2) becomes

$$
V_{x y}+c=(1 / 6) V_{x y}^{3}-V_{1}^{2} V_{x y}+1+V_{1}^{2}+(2 / 3) V_{1}^{3}
$$


If $c=.1$, then $V_{1}=.5528$ and $V_{x y}=1.2730$. The optimal rule is to accept any one offer greater than $V_{x y}-V_{1}=.7202$. Otherwise, accept both offers if the sum is greater than $V_{x y}=1.2730$. Once one object has been sold, accept any offer for the other greater than $V_{1}=.5528$.

For $1 / 2 \leq c \leq 1$, the integral in (2) over A1 and A2 is zero, while the integral over $\mathrm{A} 3$ is $V_{x y}^{3} / 2$ and the integral over $\mathrm{A} 4$ is $1-(1 / 3) V_{x y}^{3}$. The resulting equation is

$$
V_{x y}+c=(1 / 6) V_{x y}^{3}+1 .
$$

An interesting phenomenon occurs in this case. Namely, that if we were selling each object separately, we would accept the first offer that comes in. Here, in selling two objects, we would refuse both offers if the sum of the offers is less than $V_{x y}$.

Finally, if $c \geq 1$, we accept the first offers that come in and the value is $V_{x y}=1-c$.

$\S 2.2$ Dependence. It is of interest to see what effect dependence of the variates has on the optimal rules and the expected return. We look at two cases, with $X$ and $Y \in \mathcal{U}(0,1)$ having perfect positive dependence and perfect negative dependence. The conclusion is that positive dependence tends to improve the optimal return and negative dependence to worsen it; however, we have no general results along these lines.

Case 1: Perfect positive dependence, $Y=X$. Clearly, we accept both or neither. Thus, the problem is as if there was one object to sell, the cost is divided by 2 and the optimal return is multiplied by 2. For $c \leq 1$, the value is $V_{x y}=2(1-\sqrt{c}$ ) (for $k$ objects with perfect positive dependence it would be $k(1-\sqrt{2 c / k})$, and the optimal rule is to sell both objects if their common value is at least $1-\sqrt{c}$. For $c=.1$, we have $V_{x y}=1.3675$.

Case 2: Perfect negative dependence, $Y=1-X$. The optimality equation (2) becomes, $V_{x y}=\mathrm{E} \max \left\{1, X+V_{1}, 1-X+V_{1}, V_{x y}\right\}-c$. If $c \leq 1 / 8$, then $V_{1} \geq 1 / 2$ and the first option of selling both objects is never taken. This becomes the multiple house selling problem of Saario and Sakaguchi (1990) in which the objects are always sold separately and we have $V_{x y}=V_{1}+1-\sqrt{c}$. For $c=.1$, this reduces to $V_{x y}=1.2366$. For $1 / 8<c \leq 1 / 2$ $\left(0 \leq V_{1}<1 / 2\right)$, if one object has an offer of at least $1-V_{1}$, we sell just that object; otherwise we sell both objects. If $c>1 / 2\left(V_{1}<0\right)$, we always sell both objects. 
$\S$ 3. Selling Many Objects. Suppose that there are $k$ objects to be sold and let the vectors of offers be denoted by $\mathbf{X}_{1}, \mathbf{X}_{2}, \ldots$ It is assumed that these random vectors are i.i.d. copies of a random vector $\mathbf{X}=\left(X_{1}, \ldots, X_{k}\right)$ with finite second moments. Let $K=\{1,2, \ldots, k\}$ denote the set of objects to be sold. For a subset $S \subset K$, we let $V(S)$ denote the expected return using an optimal policy for selling the objects in $S$. If $S$ consists of a single point, then $V(S)$ is found as in Equation (1). If $S$ consists of two points, then $V(S)$ may be found from Equation (2). The following theorem states that the main result of the previous section still holds, and the values, $V(S)$, may be found by induction. Let $S^{c}$ denote the complement of the set $S$ in $K$ and let $V(\emptyset)=0$ where $\emptyset$ denotes the empty set.

Theorem 3. In the problem of selling $k$ objects with i.i.d. offers having finite second moments, an optimal rule exists and the value, $V(K)$, may be found inductively as the unique solution of the equation,

$$
V(K)=\mathrm{E} \max _{\emptyset \subseteq S \subseteq K}\left\{\sum_{i \in S} X_{i}+V\left(S^{c}\right)\right\}-c .
$$

The proof is an extension of the proof of Theorem 1.

$\S 3.1$ Description of the Optimal Rule. From (5), we can obtain the form of the optimal strategy. If $\mathbf{X}_{n}$ is written as $\left(X_{1, n}, \ldots, X_{k, n}\right)$ and if at stage $n, K$ represents the set of unsold objects, then the optimal rule given by the principle of optimality is to sell the objects in some subset $S_{0}$ for which $\sum_{i \in S_{0}} X_{i, n}+V\left(S_{0}^{c}\right)=\max _{\emptyset \subseteq S \subseteq K}\left\{\sum_{i \in S} X_{i, n}+V\left(S^{c}\right)\right\}$. If this maximum occurs for $S_{0}=K$, then all objects are sold and we stop. After the objects in $S_{0}$, if any, are sold, we continue to the next stage using an optimal policy for selling the objects in $S_{0}^{c}$. This continues until all objects are sold.

We may simplify this description of the optimal policy but we require the following generalization of Lemma 1. We are indebted to Fred Delbaen for the proof. The conclusion is that the value function, $V(S)$, satisfies (7) below. In game theory, such value functions are called convex and play a role in the theory of the core. See Shapley (1971).

This lemma is stated in somewhat greater generality than is needed for this paper. In particular, it is not necessary to assume that the vectors $\mathbf{X}_{1}, \mathbf{X}_{2}, \ldots$ are independent or 
identically distributed. This will be important in the extension of these results to adaptive problems in which the distribution of the $\mathbf{X}$ 's is only partially known.

A stopping vector for this problem is a vector, $\tau=\left(\tau_{1}, \ldots, \tau_{k}\right)$, of stopping rules $\tau_{i}$, each adapted to the same sequence of $\sigma$-fields, $\left\{\mathcal{F}_{n}\right\}_{n=1}^{\infty}$, where $\mathcal{F}_{n}$ contains the $\sigma$-field generated by $\mathbf{X}_{1}, \ldots, \mathbf{X}_{n}$. (This definition requires, for example, that once an object is sold, we may still observe the future offers for it.) Let $T$ denote the set of all stopping vectors. Then for a set $A \subseteq K$, the value is defined as

$$
V(A)=\sup _{\tau \in T} \mathrm{E}\left(\sum_{j \in A} X_{j, \tau_{j}}-c \max \left(\tau_{j}: j \in A\right)\right) .
$$

The only assumptions on the $\mathbf{X}_{i}$ that we use is that the expectation in (6) exists for all $\tau \in T$ and $A \subseteq K$ and that $V(S)<\infty$ for all $S$ in $K$.

Lemma 2. For arbitrary $A \subseteq K$ and $B \subseteq K$,

$$
V(A)+V(B) \leq V(A \cup B)+V(A \cap B)
$$

Proof. (F. Delbaen, private communication, 1994) Let $a_{1}, a_{2}, \ldots, a_{k}$ and $b_{1}, b_{2}, \ldots, b_{k}$ be real numbers. Then

$$
\begin{aligned}
\max & \left\{a_{1}, a_{2}, \ldots, a_{k}\right\}+\max \left\{b_{1}, b_{2}, \ldots, b_{k}\right\} \\
& \geq \max \left\{a_{1} \vee b_{1}, a_{2} \vee b_{2}, \ldots, a_{k} \vee b_{k}\right\}+\max \left\{a_{1} \wedge b_{1}, a_{2} \wedge b_{2}, \ldots, a_{k} \wedge b_{k}\right\} .
\end{aligned}
$$

To see this, let $a^{*}$ and $b^{*}$ denote the maximum of the $a_{j}$ 's and $b_{j}$ 's respectively, and suppose first that $a^{*} \geq b^{*}$. The inequality (8) reads $a^{*}+b^{*} \geq a^{*}+\max \left\{a_{1} \wedge b_{1}, a_{2} \wedge b_{2}, \ldots, a_{k} \wedge b_{k}\right\}$, or equivalently, $\max \left\{a_{1} \wedge b_{1}, a_{2} \wedge b_{2}, \ldots, a_{k} \wedge b_{k}\right\} \leq b^{*}$, which is evident. The case $a^{*} \leq b^{*}$ follows from symmetry.

Now let $\sigma=\sigma(A)$ and $\tau=\tau(B)$ be $\epsilon$-optimal stopping vectors for $A$ and $B$ respectively. Now the value of $\sigma$ does not depend on the components $\sigma_{j}$ for $j \notin A$. We extend the definition by putting $X_{j, 0}=0$ for all $j \in K$ and changing $\sigma_{j}=0$ (resp. $\tau_{j}=0$ ) for all $j \notin A$ (resp. $j \notin B$ ). Then $V(A)+V(B)$ may be written

$$
\begin{aligned}
V(A) & +V(B) \leq \mathrm{E}\left(\sum_{j \in K}\left(X_{j, \tau_{j}}+X_{j, \sigma_{j}}\right)-c\left(\max \left\{\tau_{1}, \ldots, \tau_{k}\right\}+\max \left\{\sigma_{1}, \ldots, \sigma_{k}\right\}\right)+2 \epsilon\right. \\
& =\mathrm{E}\left(\sum_{j \in K}\left(X_{j, \tau_{j} \vee \sigma_{j}}+X_{j, \tau_{j} \wedge \sigma_{j}}\right)-c\left(\max \left\{\tau_{1}, \ldots, \tau_{k}\right\}+\max \left\{\sigma_{1}, \ldots, \sigma_{k}\right\}\right)+2 \epsilon .\right.
\end{aligned}
$$


Now use the inequality (8) for the sum of the maxima in the $c$-term. Note that $\alpha_{j}:=\tau_{j} \vee \sigma_{j}$ and $\beta_{j}:=\tau_{j} \wedge \sigma_{j}$ are again stopping times vanishing on $(A \cup B)^{c}$ and $(A \cap B)^{c}$ respectively. Since they are suboptimal on $A \cup B$ and $A \cap B$ respectively, we immediately obtain

$$
V(A)+V(B) \leq V(A \cup B)+V(A \cap B)+2 \epsilon
$$

for all $\epsilon>0$. This gives inequality (7).

We remark that this proof shows the slightly stronger result that inequality (7) holds even if $V(\emptyset)$ were defined to be $-c$.

Theorem 4. Suppose at stage $n, K$ represents the set of unsold objects. Let $\mathcal{S}_{m}$ be the class of subsets of $K$ of size $m$. Let

$$
W_{m}=\max _{S \in \mathcal{S}_{m}}\left[\sum_{i \in S} X_{n, i}+V\left(S^{c}\right)\right]
$$

and let $S_{m}$ be any element of $\mathcal{S}_{m}$ achieving equality in (9). The optimal rule may be described as follows. If $W_{m}<V(K)$ for all $m$, sell no objects and go to the next stage. Otherwise, find the smallest $m>0$ such that $W_{m}>V(K)$ and sell the objects in $S_{m}$. After selling the objects in $S_{m}$ revert to this rule for selling the objects in $S_{m}^{c}$, if any, before looking at the next vector, $\mathbf{X}_{n+1}$.

Proof. Let $m$ and $S_{m}$ be as described in the theorem and let $T \subseteq K$. We will show that selling the objects in $T \cup S_{m}$ is at least as profitable as selling the objects in $T$. Then in any case it is optimal to sell the objects in $S_{m}$. If $S_{m} \subseteq T$, we are done. So assume $S_{m} \not \subset T$. Then $\left|T \cap S_{m}\right|<m$, so from the definition of $m$, we have $\sum_{i \in T \cap S_{m}} X_{i}+V\left(\left(T \cap S_{m}\right)^{c}\right) \leq$ $\sum_{i \in S_{m}} X_{i}+V\left(S_{m}^{c}\right)$. Hence,

$$
\begin{aligned}
\sum_{i \in T} X_{n, i}+V\left(T^{c}\right) & =\sum_{i \in T \cap S_{m}} X_{n, i}+\sum_{i \in T-S_{m}} X_{n, i}+V\left(T^{c}\right) \\
& \leq \sum_{i \in S_{m} \cup T} X_{n, i}+V\left(T^{c}\right)+V\left(S_{m}^{c}\right)-V\left(T^{c} \cup S_{m}^{c}\right) \\
& \leq \sum_{i \in T \cup S_{m}} X_{n, i}+V\left(T^{c} \cap S_{m}^{c}\right) \quad \text { from Lemma } 2 \\
& =\sum_{i \in T \cup S_{m}} X_{n, i}+V\left(\left(T \cup S_{m}\right)^{c}\right) .
\end{aligned}
$$


We may also use Lemma 2 to simplify the description of the sets, $A(S)$, of points in the sample space for which it is optimal to sell the objects in $S$ and none of the objects in $S^{c}$. From the principle of optimality,

$$
A(S)=\left\{\sum_{i \subset S} X_{i}+V\left(S^{c}\right) \geq \sum_{i \in T} X_{i}+V\left(T^{c}\right) \quad \text { for all } T \neq S\right\}
$$

The number of inequalities in this representation of the set $A(S)$ is $2^{k}-1$.

In the following theorem, a representation of $A(S)$ is given that uses only $\left(2^{m}-1\right)+$ $\left(2^{k-m}-1\right)$ inequalities, where $m=|S|$. We show that $A(S)$ is exactly the set of points

in the sample space for which the sale of any subset of objects of $S$ contributes at least as much its or their expected contribution (increment) of gain under an optimal policy later on, and for which, at the same time, all other subsets completely in the complement of $S$ are worth keeping unsold, since their expected contribution to the future expected gain under an optimal sales policy is larger than their present contribution.

\section{Theorem 5.}

$$
\begin{aligned}
A(S)=\{ & \sum_{i \in T} X_{i} \geq V\left(T \cup S^{c}\right)-V\left(S^{c}\right) \quad \text { for all } \emptyset \neq T \subseteq S \text { and } \\
& \left.\sum_{i \in T} X_{i} \leq V\left(S^{c}\right)-V\left(S^{c}-T\right) \quad \text { for all } \emptyset \neq T \subseteq S^{c}\right\} .
\end{aligned}
$$

Proof. First suppose $\mathbf{X}$ satisfies the inequalities of (11). Consider an arbitrary $T \subseteq K$, $T \neq S$ and cancel $X_{i}$ for $i \in S \cap T$ from both sides of equation (10). We are to show

$$
\sum_{i \in T-S} X_{i}+V\left(T^{c}\right) \leq \sum_{i \in S-T} X_{i}+V\left(S^{c}\right)
$$

Since $T-S \subset S^{c}$ and $S-T \subset S$, we have from (11)

$$
\begin{aligned}
\sum_{i \in T-S} X_{i}+V\left(T^{c}\right) & \leq V\left(S^{c}\right)-V\left(S^{c} \cap(T-S)^{c}\right)+V\left(T^{c}\right) \\
& =V\left(S^{c}\right)-V\left(S^{c} \cap T^{c}\right)+V\left(T^{c}\right) \\
& \leq V\left(S^{c} \cup T^{c}\right)=V\left((S-T) \cup S^{c}\right) \\
& \leq \sum_{i \in S-T} X_{i}+V\left(S^{c}\right)
\end{aligned}
$$


as was to be shown.

Now suppose $\mathbf{X}$ satisfies the inequalities of (10). Then for $T \subseteq S$,

$$
\sum_{i \in T} X_{i}=\sum_{i \in S} X_{i}-\sum_{i \in S-T} X_{i} \geq V\left((S-T)^{c}\right)-V\left(S^{c}\right)=V\left(S^{c} \cup T\right)-V\left(S^{c}\right),
$$

and for $T \subseteq S^{c}$

$$
\sum_{i \in T} X_{i}=-\sum_{i \in S} X_{i}+\sum_{i \in S \cup T} X_{i} \leq V\left(S^{c}\right)-V\left((T \cup S)^{c}\right)=V\left(S^{c}\right)-V\left(S^{c}-T\right)
$$

§3.2. Selling $k$ identical objects. In the case of $k$ identical objects with i.i.d. offers, the description of the optimal rule is easy. Let the offers be denoted by $X_{1}, \ldots, X_{k}$. (Independence of $X_{1}, \ldots, X_{k}$ is not required for this result; exchangeability suffices.) Let $V_{j}$ represent the value of the problem with $j$ identical objects (e.g. $V_{x y}=V_{x z}=V_{2}$ etc.) and let $\left.V_{0}=0\right)$. The optimality equations become, inductively in $k$,

$$
V_{k}=\mathrm{E}\left\{\max _{0 \leq j \leq k} \max _{S \in \mathcal{S}_{j}}\left[\left(\sum_{i \in S} X_{i}\right)+V_{k-j}\right]\right\}-c
$$

where $\mathcal{S}_{j}$ is the set of all subsets of size $j$ in $\{1,2, \ldots, k\}$. The optimal rule is as follows. Order the offers in decreasing order, $X_{1} \geq X_{2} \geq \ldots \geq X_{k}$. If $X_{1} \geq V_{k}-V_{k-1}$, accept $X_{1}$. Otherwise, if $X_{1}+X_{2} \geq V_{k}-V_{k-2}$, accept $X_{1}$ and $X_{2}$. Otherwise, ... Otherwise, if $X_{1}+\cdots+X_{k} \geq V_{k}$, accept all offers. Otherwise accept no offers. Once any objects are sold, revert to the optimal strategy for selling the remaining objects (before looking at the next vector of offers).

The argument is straightforward and does not even use convexity. With the $X_{i}$ arranged in decreasing order, the inside maximum in (12) occurs at $X_{1}+\cdots+X_{j}+V_{k-j}$ for some $j$. If it is optimal to accept anything, it is optimal to accept at least $X_{1}$. So, if $X_{1} \geq V_{k}-V_{k-1}$, it is optimal to accept $X_{1}$. Similarly, if $X_{1}<V_{k}-V_{k-1}$ but $X_{1}+X_{2} \geq V_{k}-V_{k-2}$, it is optimal to select both $X_{1}$ and $X_{2}$ at least. And so forth.

$\S 3.3$ Example. As an illustration, consider the case $k=3$ with independent uniform $(0,1)$ random variables. We solve the optimality equations (12) for $V_{1}, V_{2}$ and $V_{3}$. In Example 2.1, the values of $V_{1}$ and $V_{2}$ were found as functions of $c$. The expectation in 
(12) breaks down into eight regions, as in Theorem 5, but only four have distinct values because the variables are exchangeable. Through straightforward but tedious integration separately over these regions, we may find the expectation in (12) separately for four cases.

For $0<c \leq 1 / 2$, the expectation in $(12)$ is

$$
\begin{aligned}
\frac{3}{2}+\frac{3}{2} V_{1}^{2}+2 V_{1}^{3} & +\frac{3}{8} V_{1}^{4}-3 V_{1}^{2} V_{2}-\frac{3}{2} V_{1}^{2} V_{2}^{2}+\frac{1}{2} V_{2}^{3}+\frac{3}{8} V_{2}^{4}-V_{1}^{3} V_{3} \\
& +3 V_{1}^{2} V_{2} V_{3}-\frac{1}{2} V_{2}^{3} V_{3}-\frac{3}{4} V_{1}^{2} V_{3}^{2}+\frac{1}{24} V_{3}^{4} .
\end{aligned}
$$

Setting this to $V_{3}+c$ and solving for $V_{3}$ gives the optimal return. When $c=.1$, we have $V_{1}=.5528, V_{2}=1.2730$, and we find $V_{3}=2.0354$. See Table 1 .

For $.5 \leq c \leq 1$, we have $V_{1}<0$, so that (12) reduces to $V_{3}=\operatorname{Emax}\{X+Y+Z, X+$ $\left.V_{2}, Y+V_{2}, Z+V_{2}, V_{3}\right\}-c$. For $1 \leq c \leq 1.5$, we have $V_{3}=\mathrm{E} \max \left(X+Y+Z, V_{3}\right)-c$, or equivalently, $\mathrm{E}\left(X+Y+Z-V_{3}\right)^{+}=c$. This reduces to the equation, $V_{3}^{4}-24 V_{3}+36-24 c=0$. For $c>1.5, V_{3}=1.5-c$.

Table 1. Values of $V_{1}, V_{2}$, and $V_{3}$ for independent uniform $(0,1)$ offers and various values of $c$.

$\begin{array}{ccccccccccccccc}c & .1 & .2 & .3 & .4 & .5 & .6 & .7 & .8 & .9 & 1.0 & 1.1 & 1.2 & 1.3 & 1.4 \\ V_{1} & .553 & .368 & .225 & .106 & 0 & -.1 & -.2 & -.3 & -.4 & -.5 & -.6 & -.7 & -.8 & -.9 \\ V_{2} & 1.273 & 1.000 & .804 & .651 & .524 & .412 & .305 & .201 & .100 & 0 & -.1 & -.2 & -.3 & -.4 \\ V_{3} & 2.035 & 1.679 & 1.428 & 1.235 & 1.079 & .946 & .825 & .713 & .606 & .503 & .401 & .300 & .200 & .100\end{array}$

$\S 4$. Selling the objects with recall. Suppose we are able to accept any of the past offers. The setup is as follows. We observe $\mathbf{X}_{1}, \mathbf{X}_{2}, \ldots$ sequentially, assumed to be i.i.d. $k$-dimensional random vectors with finite second moments. We may observe as long as we please at a cost of $c>0$ per observed vector, and when we stop we may select the largest offer for each object from among all past offers. Thus our payoff if we stop at stage $n$ is, $\sum_{i=1}^{k} M_{n}^{(i)}-n c$, where

$$
M_{n}^{(i)}=\max \left\{X_{1}^{(i)}, \ldots, X_{n}^{(i)}\right\} \quad \text { for } \quad i=1, \ldots, k
$$


Theorem 6. In the problem of selling $k \geq 1$ objects with recall, based on i.i.d. vector offers having finite second moments, the stopping rule

$$
N_{1}=\min \left\{n \geq 1: \sum_{j=1}^{k} \phi_{j}\left(M_{n}^{(j)}\right) \leq c\right\}
$$

is optimal, where $\phi_{j}(x)=\mathrm{E}\left(X^{(j)}-x\right)^{+}$for $j=1, \ldots, k$.

Proof. We show that $N_{1}$ is the optimal rule by showing it is the one-stage look-ahead rule and showing the problem is monotone. If we are at stage $n$ and stop, we would receive $M_{n}^{(1)}+\cdots+M_{n}^{(k)}-n c$. If we continue one stage and stop, we expect to receive

$$
\mathrm{E}\left[\max \left\{M_{n}^{(1)}, X_{n+1}^{(1)}\right\}+\cdots+\max \left\{M_{n}^{(k)}, X_{n+1}^{(k)}\right\}-(n+1) c \mid \mathcal{F}_{n}\right]
$$

where $\mathcal{F}_{n}$ represents the sigma-field generated by the first $n$ vectors. The one-stage lookahead rule calls for stopping as soon as the former is greater than the latter. Namely, we stop at the first $n$ such that

$$
M_{n}^{(1)}+\cdots+M_{n}^{(k)} \geq \mathrm{E}\left[\max \left\{M_{n}^{(1)}, X_{n+1}^{(1)}\right\}+\cdots+\max \left\{M_{n}^{(k)}, X_{n+1}^{(k)}\right\}-(n+1) c \mid \mathcal{F}_{n}\right]-c
$$

Thus, the one-stage look-ahead rule stops at the first $n$ for which

$$
\sum_{j=1}^{k} \phi_{j}\left(M_{n}^{(j)}\right) \leq c .
$$

Since we have assumed finite second moments, the one-stage look-ahead rule is optimal provided the problem is monotone. To show the problem is monotone, we must show that if the one-stage look-ahead rule calls for stopping at stage $n$ then it will call for stopping with probability one at all future stages. Since the $\phi_{j}(x)$ are non-increasing in $x$, we see that the problem is monotone since $M_{n}^{(1)}, \ldots, M_{n}^{(k)}$ are almost surely nondecreasing.

It is of interest to note that the optimal rule depends on the joint distribution of the offers only through the marginal distributions.

As an example, suppose the $X^{(j)}$ have marginal uniform distributions on the interval $(0,1)$. The functions $\phi_{j}$ become $\phi_{j}(x)=(1-x)^{2} / 2$, and the optimal rule is to stop at the first $n$ for which

$$
\left(M_{n}^{(1)}-1\right)^{2}+\cdots+\left(M_{n}^{(k)}-1\right)^{2} \leq 2 c .
$$


Thus, we stop when the Euclidean distance from $\left(M_{n}^{(1)}, \ldots, M_{n}^{(k)}\right)$ to $(1, \ldots, 1)$ is at most $\sqrt{2 c}$. If the $X^{(j)}$ have marginal exponential distributions with mean 1 , the optimal rule is to stop at the first $n$ for which

$$
\exp \left\{-M_{n}^{(1)}\right\}+\cdots+\exp \left\{-M_{n}^{(k)}\right\} \leq c .
$$

$\S 5$. Discounted problems. The extension of this multiple selling problem to the case without a cost but with a discount, $0<\beta<1$, can be similarly treated, but we cannot carry the solution as far. For simplicity we treat the model without recall only in the case $k=2$ of two objects. A model with recall is treated in Section 5.3. Let $\left(X_{1}, Y_{1}\right),\left(X_{2}, Y_{2}\right), \ldots$, be a sequence of i.i.d. 2-dimensional random vectors with finite first moments. Finite second moments are not needed in the discounted problems we treat.

The simplest extension is to allow discounting on each object until it is sold. This leads to an optimality equation of the form,

$$
V_{x y}=\beta \mathrm{E} \max \left\{X+Y, X+V_{y}, V_{x}+Y, V_{x y}\right\},
$$

where $V_{y}$ and $V_{x}$ are the optimal returns for selling the $y$ and $x$ objects optimally with discount $\beta$, and satisfy the equations determined by Karlin (1962),

$$
V_{x}=\beta \mathrm{E} \max \left\{X, V_{x}\right\} \quad \text { and } \quad V_{y}=\beta \mathrm{E} \max \left\{Y, V_{y}\right\}
$$

For this formulation, it is optimal to sell the objects optimally separately, and $V_{x y}=$ $V_{x}+V_{y}$. The possible dependence of $X$ and $Y$ plays no role in this result.

To obtain a nontrivial result analogous to the cost case, two modifications of this model are presented. The more direct extension to an additive model to selling many objects does not seem to be as flexible as was possible for the cost model. Therefore a multiplicative model is also introduced.

$\S 5.1$ Additive model. We assume that if one object is sold, the discount on its value continues until the other is sold. In other words, money from the first sale cannot be deposited in the bank until the second object is sold as well. Thus, if one object is sold 
at a value $X=x$ (respectively $Y=y$ ), continuing optimally to sell the other object gives a return $V_{y}(x)$ (respectively $\left.V_{x}(y)\right)$ satisfying

$$
V_{y}(x)=\beta \mathrm{E} \max \left\{Y+x, V_{y}(x)\right\} \quad \text { and } \quad V_{x}(y)=\beta \mathrm{E} \max \left\{X+y, V_{x}(y)\right\}
$$

It is useful to rearrange terms and rewrite this in the form

$$
\frac{(1-\beta)}{\beta} V_{y}(x)=\mathrm{E}\left(Y+x-V_{y}(x)\right)^{+} \quad \text { and } \quad \frac{(1-\beta)}{\beta} V_{x}(y)=\mathrm{E}\left(X+y-V_{x}(y)\right)^{+},
$$

corresponding to (1). The optimality equation for the problem of selling the objects together then becomes

$$
V_{x y}=\beta \mathrm{E} \max \left\{X+Y, V_{y}(X), V_{x}(Y), V_{x y}\right\}
$$

corresponding to (2). In the modeling of this problem as a buying problem, we must travel from store to store until both objects are bought. Then we return home and the discounting stops.

It seems difficult to give general reductions on the form of the optimal rule for this problem. Instead, we solve it in the special case of independent uniform distributions on the interval $(0,1)$. In this case, $V_{x}(z)=V_{y}(z)$ for all $z$ so we may drop the subscript. Since clearly $V(x)-x<1$, equation (14) reduces to

$$
\begin{aligned}
\frac{(1-\beta)}{\beta} V(z) & =\mathrm{E}(X+z-V(z))^{+} \\
& =\int_{(V(z)-z)^{+}}^{1}(x+z-V(z)) d x \\
& = \begin{cases}(1-V(z)+z)^{2} / 2 & \text { if } V(z) \geq z \\
\frac{1}{2}-V(z)+z & \text { if } V(z)<z\end{cases}
\end{aligned}
$$

and leads for $V(z) \geq z$ to the equation

$$
V(z)^{2}-2 V(z)\left(z+\frac{1}{\beta}\right)+(1+z)^{2}=0,
$$

with solution,

$$
V(z)= \begin{cases}z+\frac{1}{\beta}-\frac{1}{\beta} \sqrt{1-\beta^{2}+2 z \beta(1-\beta)} & \text { if } z \leq \beta /(2(1-\beta)) \\ \beta\left(\frac{1}{2}+z\right) & \text { if } z>\beta /(2(1-\beta)\end{cases}
$$


For $\beta \geq 2 / 3$, we have $\beta /(2(1-\beta))>1$ so the lower half of this expression is not in force. From this, (15) reduces to

$$
\begin{aligned}
V_{x y}=\beta \mathrm{E} \max \{X+Y, & X+\frac{1}{\beta}-\frac{1}{\beta} \sqrt{1-\beta^{2}+2 X \beta(1-\beta)}, \\
Y & \left.+\frac{1}{\beta}-\frac{1}{\beta} \sqrt{1-\beta^{2}+2 Y \beta(1-\beta)}, V_{x y}\right\} .
\end{aligned}
$$

One may find $V_{x y}$, by numerical integration coupled with some root finding procedure. To give an indication of the size of the optimal expected payoff, this equation was solved or several values of $\beta$. If $V_{x y}(\beta)$ denotes this value, we find $V_{x y}(.8)=.9181, V_{x y}(.9)=1.1643$, and $V_{x y}(.95)=1.3673$.

$\S 5.2$ Multiplicative Model. As another extension to a discounted version of this problem we consider maximizing the product rather than the sum of the values. For this version we assume the offers for the objects are nonnegative, $X \geq 0$ and $Y \geq 0$, and that the payoff is the product of $X$ and $Y$. This could be considered as an assignment problem where $X$ represents the value of the job and $Y$ the ability of the worker, similar to a model treated by Derman, Lieberman and Ross (1972), except that in our model both workers and jobs arrive. A model for matching pairs of arrivals may be found in the paper of David and Yechiali (1986).

The optimality equation for discount $\beta$ becomes

$$
V_{x y}=\beta \mathrm{E} \max \left\{X Y, V_{y}(X), V_{x}(Y), V_{x y}\right\}
$$

where $V_{x}(y)$ and $V_{y}(x)$ are the optimal return functions for the maximizing $X y$ and $Y x$ respectively. These functions satisfy the equations

$$
V_{x}(y)=\beta \operatorname{E} \max \left\{X y, V_{x}(y)\right\} \quad \text { and } \quad V_{y}(x)=\beta \operatorname{E} \max \left\{Y x, V_{y}(x)\right\} .
$$

From this, we can see that $V_{y}(x)=x V_{y}$, where $V_{y}$ is the unique number satisfying $V_{y}=$ $\beta \mathrm{E} \max \left\{Y, V_{y}\right\}$ and similarly $V_{x}(y)=y V_{x}$, where $V_{x}$ is the unique number satisfying $V_{x}=$ $\beta \mathrm{E} \max \left\{X, V_{x}\right\}$. The optimality equation reduces to

$$
V_{x y}=\beta \mathrm{E} \max \left\{X Y, X V_{y}, Y V_{x}, V_{x y}\right\} .
$$

It is not difficult to show using the method of proof of Theorem 1 that there is a unique solution to this equation for $V_{x y}$ and that the principle of optimality leads to an optimal rule. When $X$ and $Y$ are independent, the description of this rule may be simplified using the following analog of Lemma 1. 
Lemma 4. If $X$ and $Y$ are independent, the value function is logarithmically superadditive. In fact, $V_{x} V_{y} \leq \beta V_{x y}$.

Proof. Let $\sigma$ and $\tau$ be optimal rules satisfying $V_{x}=\mathrm{E} \beta^{\sigma} X_{\sigma}$ and $V_{y}+\mathrm{E} \beta^{\tau} Y_{\tau}$, where $\sigma$ depends on $X_{1}, X_{2}, \ldots$ only and $\tau$ depends on $Y_{1}, Y_{2}, \ldots$ only. Then $\sigma$ and $\tau$ are independent, and

$$
\begin{aligned}
V_{x} V_{y} & +\mathrm{E} \beta^{\sigma+\tau} X_{\sigma} Y_{\tau} \\
& \leq \beta \mathrm{E} \beta^{\max (\sigma, \tau)} X_{\sigma} Y_{\tau} \\
& \leq \beta V_{x y} .
\end{aligned}
$$

We do not know if the logarithmic convexity analogue of Lemma 2 holds for this model of selling more than two assets.

Logarithmic superadditivity implies that the optimal selling regions have a form similar to that of Figure 1 but with the line $x+y=V_{x y}$ replaced by the curve $x y=V_{x y}$.

As an example, consider the case of $X$ and $Y$ independent uniform random variables on the interval $(0,1)$. Then,

$$
V_{1}=V_{x}=V_{y}=\frac{1}{\beta}-\sqrt{\frac{1}{\beta^{2}}-1} .
$$

The expectations of $\max \left\{X Y, X V_{1}, Y V_{1}, V_{x y}\right\}$ over the four regions corresponding to those of Figure 1 becomes

$$
\begin{aligned}
& \mathrm{A} 1:\left(V_{1}^{2}-V_{x y}^{2}\right) / 2 \\
& \mathrm{~A} 2:\left(V_{1}^{2}-V_{x y}^{2}\right) / 2 \\
& \mathrm{~A} 3: V_{x y}^{2}\left(1+\ln \left(V_{x y} / V_{1}\right)\right) \\
& \mathrm{A} 4:\left(1-2 V_{1}^{2}+V_{x y}^{2}\right) / 4-\left(V_{x y}^{2} / 2\right) \ln \left(V_{x y} / V_{1}^{2}\right)
\end{aligned}
$$

From this, the optimality equation (16) becomes

$$
V_{x y}=\beta\left[\frac{1}{4}\left(1+2 V_{1}^{2}+V_{x y}^{2}\right)+\frac{V_{x y}^{2}}{2} \ln \left(V_{x y} / V_{1}^{2}\right)\right]
$$

This root of this equation is easily found by iteration. If $V_{1}(\beta)$ and $V_{x y}(\beta)$ denote the values, then $V_{1}(.8)=.5, V_{x y}(.8)=.3359, V_{1}(.9)=.6268, V_{x y}(.9)=.4686, V_{1}(.95)=.7239$, and $V_{x y}(.95)=.5866$. 
$\S 5.3$ With Recall. In the discounted case, the problem of selling the objects with recall has a solution quite similar to that of Section 4 for the cost case. In the notation of that section, the payoff for stopping at stage $n$ is $\beta^{n} \sum_{i=1}^{n} M_{n}^{(i)}$. The one-stage look-ahead rule has a form similar to that of Theorem 6, namely,

$$
N_{1}=\min \left\{n \geq 1: \sum_{j=1}^{k} \phi_{j}\left(M_{n}^{(j)}\right) \leq \frac{1-\beta}{\beta} \sum_{j=1}^{k} M_{n}^{(j)}\right\},
$$

where as before $\phi_{j}(x)=\mathrm{E}\left(X^{(j)}-x\right)^{+}$. Since the $\phi_{j}(x)$ are nonincreasing in $x$ and the $M_{n}^{(j)}$ are almost surely nondecreasing in $n$ for each $j$, the problem is monotone and so $N_{1}$ is optimal (under the assumption of finite second moments). Again, we see the optimal rule depends on the joint distribution of offers only through the marginal distributions. The details follow as in Section 4.

Acknowledgement: The authors are indebted to Fred Delbaen for the elegant proof of Lemma 2. They would also like to thank the National Science Foundation of Belgium FNRS for financial support and the referee for his/her comments.

\section{REFERENCES}

S. C. Albright (1974) Optimal sequential assignments with random arrival times, Man. Sci. 21, 60-70.

E. J. Collins and J. M. McNamara (1993) The job-search problem with competition: An evolutionarily stable dynamic strategy, Adv. Appl. Prob. 25, 314-333.

Y. S. Chow and H. Robbins (1961) A martingale system theorem and applications, Fourth Berk. Symp. on Math. Stat. \& Prob. 1 93-104.

Y. S. Chow, H. Robbins, and D. Siegmund (1971) Great Expectations: The Theory of Optimal Stopping, Houghton Mifflin, Boston.

I. David and U. Yechiali (1986) Sequential assignment match processes with arrivals of candidates and offers, Prob. Eng. Inf. Sci. 4, 413-430.

M. H. DeGroot (1970) Optimal Statistical Decisions, McGraw-Hill, New York.

C. Derman and J. Sacks (1960) Replacement of periodically inspected equipment, Naval Res. Logist. Quart. 7 597-607. 
C. Derman, G. J. Lieberman and S. M. Ross (1972) A sequential stochastic assignment problem, Man. Sci. 18, 349-355.

S. Karlin (1962) Stochastic models and optimal policy for selling an asset, Studies in Appl. Prob. and Man. Sci. Stanford Univ. Press, 148-158.

J. MacQueen and R. G. Miller (1960) Optimal persistence policies, Oper. Res. 8, 362-380.

J. McMillan and M. Rothschild (1994) Search, Chapter 24 in Handbook of Game Theory with Economic Applications 2, R. J. Aumann and S. Hart, eds., North Holland/Elsevier Publishers, New York, 905-927.

J. M. McNamara and E. J. Collins (1990) The job-search problem as an employercandidate game, J. Appl. Prob. 27, 815-827.

T. Nakai (1986) An optimal selection problem for a sequence with a random number of applicants per period, Op. Res. 34, 478-485.

R. Righter (1990) Stochastically maximizing the number of successes in a sequential assignment problem, J. Appl. Prob. 27, 351-364.

V. Saario (1986) Comparison of the discrete and continuous-time stochastic selling models, Engineering Costs and Production Economics 12, 15-20.

V. Saario and M. Sakaguchi (1990) Some generalized house-selling problems, Math. Japonica 35, 861-873.

M. Sakaguchi (1961) Dynamic programming of some sequential sampling design, J. Math. Anal. Appl. 2, 446-466.

M. Sakaguchi (1973) Optimal stopping in sampling from a bivariate distribution, J. Oper. Res. Soc. Japan 16, 186-200.

M. Sakaguchi (1978) When to stop: randomly appearing bivariate target values, J. Oper. Res. Soc. Japan 21, 45-58.

L. S. Shapley (1971) Cores of convex games, Int. J. Game Theory 1, 11-26.

W. Stadje (1985) On multiple stopping rules, Optimization 16, 410-418.

W. Stadje (1990) A full information pricing problem for the sale of several identical commodities, Zeit. Oper. Res. 34, 161-181. 
G. J. Stigler (1961) The economics of information, J. Political Economy 69 213-225.

G. J. Stigler (1962) Information in the labor market, J. Political Economy 70 Supplement Oct. 94-105. 